\title{
Research Paper \\ The Age- and Gender-wise Assessment of the Position and Shape of Mental Foramen in Patients' Panoramic Radiography
}

\author{
Hamzeh Ekran ${ }^{1}\left(\mathbb{D}\right.$, Amin Ghanbarnejad $^{2}$ (D) ${ }^{*}$ Masomeh Afsa $^{3}$ (1)
}

1. Department of Endodontics, School of Dental, Shahid Sadoughi University of Medical Sciences, Yazd, Iran.

2. Department of Biostatistics, School of Health, Hormozgan University of Medical Sciences, Hormozgan, Iran.

3. Department of Radiology, School of Dental, Hormozgan University of Medical Sciences, Hormozgan, Iran.

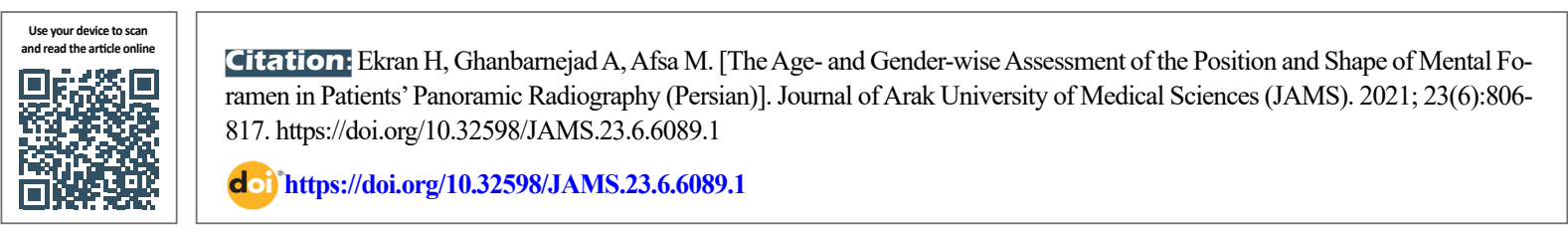

\section{(i) (3)}

Article Info:

Received: 31 Jan 2020

Accepted: 07 Aug 2020

Available Online: 01 Feb 2021

Keywords:

Mental foramen, Panoramic radiography, Position of mental foramen, Mental foramen

\section{A B STRACT}

Background and Aim Recognizing the position of the Mental Foramen (MF) is essential in numerous cases, such as anesthesia injection and periapical surgeries in the anterior region of the mandible. Furthermore, the diversity in the location and position of MF can develop problems during surgery in this region. Methods \& Materials The present study examined anatomical landmarks based on panoramic radiographic images obtained in Bandar Abbas City, Iran. In total, 450 panoramic radiographic images of men and women were assessed. All explored images were converted to JPEG format and entered in real size in Auto CAD software (2014). The collected data were analyzed in SPSS by one-way Analysis of Variance (ANOVA), Student's t-test, and Chi-squared test.

Ethical Considerations This study was approved by the Ethics Committee of Hormozgan University of Medical Sciences (Code: IR.HUMS.REC.1394.189).

Results The obtained data revealed that the mean distance from the MF to the lower edge of the mandible on the right and left was $10.53 \mathrm{~mm}$ and $10.51 \mathrm{~mm}$, respectively. The mean distance from the MF to the posterior side of the mandible equaled $49.36 \mathrm{~mm}$ on the right and $48.72 \mathrm{~mm}$ on the left. Moreover, the mean distance of MF to the midline of the lower jaw on the right and left was calculated as 27.16 and $26.27 \mathrm{~mm}$, respectively. Furthermore, in most cases, the anterior-posterior position of MF was symmetrical concerning anatomical landmarks. The anterior-posterior distance of MF to mandibular midline was significantly different between the explored males and females $(P<0.001)$. There was no significant gender-wise difference in the shape of the MF $(P=0.89)$.

Conclusion The present research results signified that the most frequent anterior-posterior position of the MF is between the apex of the first and second premolars. Furthermore, the most prevalent shape of MF is oval per panoramic images.

\section{Extended Abstract}

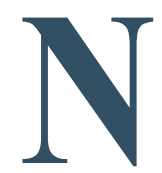

\section{Introduction}

umerous surgeries are performed on the anterior region of the lower jaw (the area between two chin holes). Furthermore, the location of the chin hole (Mental Foramen; MF) is critical in multiple cases, like anesthesia injection and periapical surgeries in the anterior lower jaw. Besides, the diversity in the location and position of this hole can generate problems during surgery in this region.

\section{* Corresponding Author:}

Masomeh Afsa, PhD.

Address: Department of Radiology, School of Dental, Hormozgan University of Medical Sciences, Hormozgan, Iran.

Tel: +98 (76) 33330458

E-mail: masomeh.afsa@hums.ac.ir 
Table 1. Age-wise Mean $\pm \mathrm{SD}$ values of the anterior-posterior distances of the mental foramen

\begin{tabular}{|c|c|c|c|c|c|c|c|c|c|c|}
\hline \multirow{3}{*}{ Variables } & \multicolumn{8}{|c|}{ Mean士SD } & \multirow{3}{*}{ P Right } & \multirow{3}{*}{ P Left } \\
\hline & \multicolumn{2}{|c|}{ Age Group A } & \multicolumn{2}{|c|}{ Age Group B } & \multicolumn{2}{|c|}{ Age Group C } & \multicolumn{2}{|c|}{ Age Group D } & & \\
\hline & Right & Left & Right & Left & Right & Left & Right & Left & & \\
\hline Distance to midline & $25.5 \pm 2.95$ & $26.4 \pm 3.26$ & $27.2 \pm 3.14$ & $26.1 \pm 3.25$ & $27.25 \pm 2.96$ & $26.41 \pm 2.81$ & $27 \pm 3.85$ & $27.2 \pm 3.08$ & 0.64 & 0.35 \\
\hline $\begin{array}{l}\text { Distance to the } \\
\text { posterior side }\end{array}$ & $48.01 \pm 5.18$ & $46.66 \pm 6.16$ & $49.59 \pm 4.53$ & $48.96 \pm 5.12$ & $49.28 \pm 4.66$ & $48.72 \pm 4.58$ & $48.9 \pm 4.28$ & $48.6 \pm 4.2$ & 0.28 & 0.09 \\
\hline $\begin{array}{l}\text { Distance to the } \\
\text { lower side }\end{array}$ & $9.9 \pm 1.32$ & $9.9 \pm 1.75$ & $10.43 \pm 1.54$ & $10.49 \pm 1.5$ & $10.72 \pm 1.56$ & $10.63 \pm 1.59$ & $11.41 \pm 1.33$ & $10.91 \pm 1.22$ & 0.001 & 0.04 \\
\hline
\end{tabular}

\section{Materials and Methods}

In this study, 450 panoramic radiographic images of men and women were evaluated. All images were converted to JPEG format and entered in real size in Auto CAD software (2014). Data analysis was performed in SPSS using oneway Analysis of Variance (ANOVA), Student's t-test, and Chi-squared tests.

\section{Results}

The mean distance from the MF to the lower edge of the mandible on the right and the left were equal to $10.53 \mathrm{~mm}$ and $10.51 \mathrm{~mm}$, respectively. The mean distance from the MF to the posterior side of the lower jaw was measured as $49.36 \mathrm{~mm}$ on the right and $48.72 \mathrm{~mm}$ on the left. Additionally, the mean distance of this hole to the midline of the lower jaw on the right and left was 27.16 and $26.27 \mathrm{~mm}$, respectively. In most explored cases, the anterior-posterior position of this hole was symmetrical concerning anatomical landmarks. The anterior-posterior distance of MF to the lower jaw midline presented significant gender-wise differences $(\mathrm{P}<0.001)$. The shape of the MF provided no genderwise significant difference $(\mathrm{P}=0.89)$ (Table $1 \& 2)$.

\section{Discussion and Conclusion}

The current research results suggested that the most frequent anterior-posterior position of the MF is located between the apex of the first and second premolars. Furthermore, the most prevalent shape of the MF using panoramic images was oval. According to the data obtained in the present study and other investigations, the shape of the MF in the offending population was mainly oval. In general, the differences in the location and position of the MF in this research, compared to other studies can be attributed to morphological and anthropological variations in various populations.

\section{Ethical Considerations}

\section{Compliance with ethical guidelines}

This study was approved by the Ethics Committee of Hormozgan University of Medical Sciences (Code: IR.HUMS. REC.1394.189).

\section{Funding}

This article is extracted from the MD. dissertation of dental dentistry of the first author at the Department of End-

Table 2. The gender-wise Mean \pm SD scores of the anterior-posterior distances of the mental foramen

\begin{tabular}{|c|c|c|c|c|c|c|}
\hline \multirow{3}{*}{ Variables } & \multicolumn{4}{|c|}{ Mean \pm SD } & \multirow{3}{*}{ P Right } & \multirow{3}{*}{ P Left } \\
\hline & \multicolumn{2}{|c|}{ Males } & \multicolumn{2}{|c|}{ Females } & & \\
\hline & Right & Left & Right & Left & & \\
\hline Distance to midline & $27.62 \pm 3.19$ & $26.68 \pm 3.19$ & $26.74 \pm 2.99$ & $25.9 \pm 3.03$ & 0.001 & 0.006 \\
\hline Distance to posterior border & $50.5 \pm 4.65$ & $49.8 \pm 4.27$ & $48.34 \pm 4.31$ & $47.76 \pm 5.43$ & $<0.0001$ & $<0.0001$ \\
\hline Distance to lower border & $11.14 \pm 1.5$ & $11.08 \pm 1.55$ & $9.98 \pm 1.35$ & $10.0 \pm 1.31$ & $<0.0001$ & $<0.0001$ \\
\hline
\end{tabular}


Table 3. Gender-wise shape of the mental foramen in panoramic radiography

\begin{tabular}{|c|c|c|c|c|}
\hline \multirow{2}{*}{ Variables } & \multicolumn{4}{|c|}{ No. (\%) } \\
\hline & \multicolumn{2}{|c|}{ The Shape of the Chin Hole on the Right Side of the Mandible } & \multicolumn{2}{|c|}{ The Shape of the Mental Foramen on the Left Side of the Mandible } \\
\hline Gender & Round & Oval & Round & Oval \\
\hline Males & $32(15.1)$ & $180(84.9)$ & $27(12.7)$ & $185(78.3)$ \\
\hline Females & $37(15.5)$ & $201(84.5)$ & $32(13.4)$ & $206(86.6)$ \\
\hline $\mathrm{P}$ & \multicolumn{2}{|c|}{0.89} & \multicolumn{2}{|c|}{0.82} \\
\hline
\end{tabular}

odontics, School of Dental, Shahid Sadoughi University of Medical Sciences, Yazd.

\section{Authors' contributions}

Conceptualization and supervision: Masoumeh Afsa; Data collection, Writing - original draft: Hamzeh Ekran; Data analysis, writing - review \& editing: Amin Ghanbarnejad.

\section{Conflicts of interest}

The authors stated no conflicts of interest.

\section{Acknowledgements}

We would like to thank all Staff of Hormozgan University of Medical Sciences and all the staff and colleagues of the School of Dentistry, Department of Radiology who contributed to this research. 
بررسى موقعيت و شكل سوراخ جانهاى بر حسب سن و جنسيث بيمار ان مراجعه كثنده به دانشيًاه علوميزشكي بندرعباس

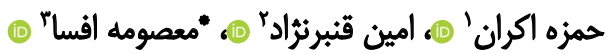

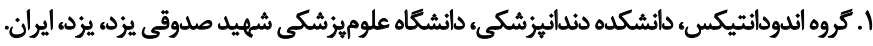

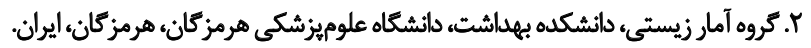

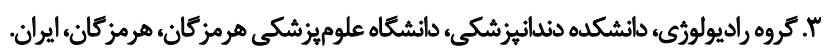

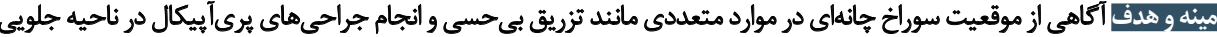

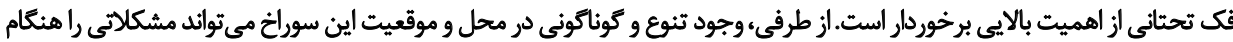

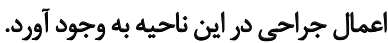

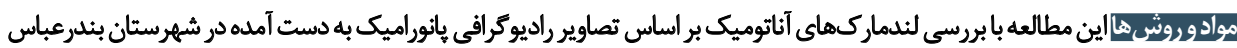

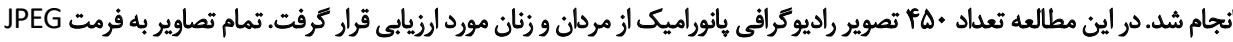

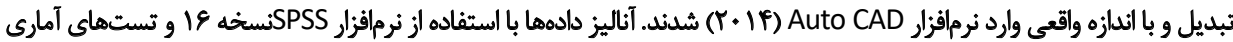
Chi Square, One way ANOVA ،Student T-test

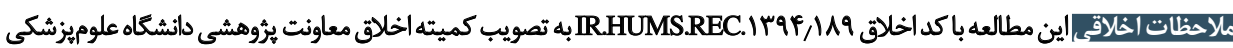
هرمزكان ريسيد.

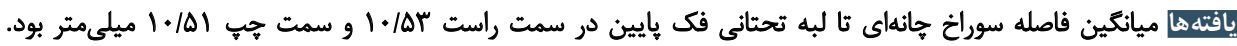

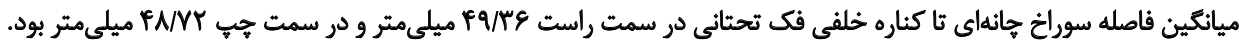

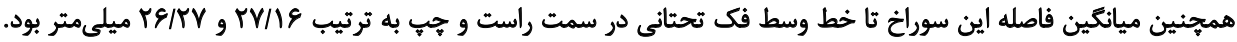

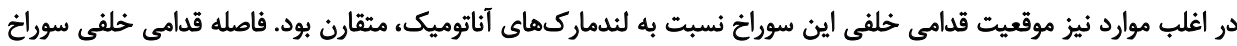

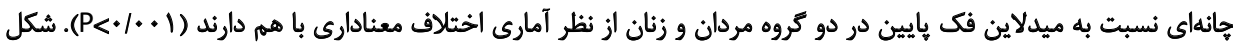

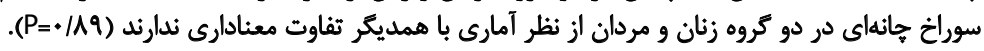

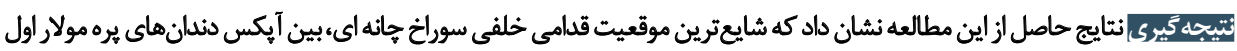

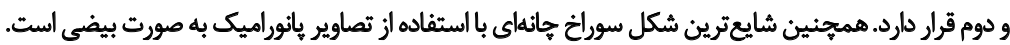

اطلاعات مقاله:

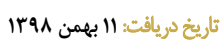

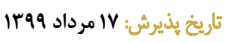
تاريخ أتشار: كا بهمن

كليدوازوها:

سوراخ جانهاي، راديوكرافى يانوراميك،

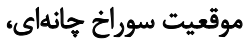
شكل سوراخ جانهاى

علاوه بر اين، يبى بردن به محل دقيق اين عصب در بازسازى الزي

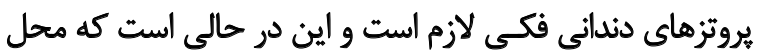

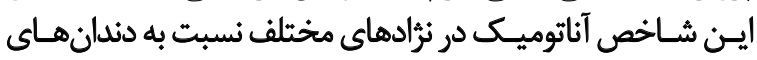

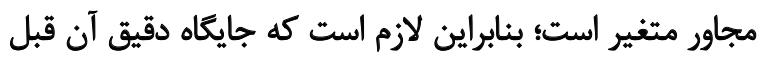
از انجام هركونه دستكارى و اعمال جراحى يا يارئ يروتزى شناخته

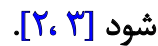

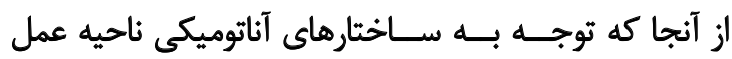

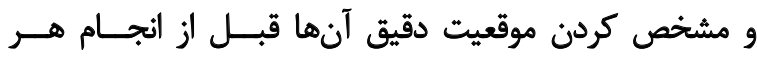

doles

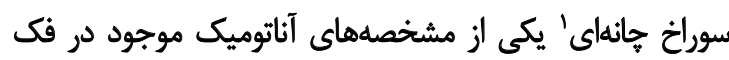

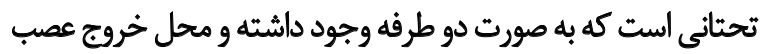

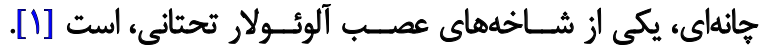
بي حس كردن عصب هانهاي در جراحىهاى متعدد دندانى فكى التى نياز است.

\section{Mental Foramen}

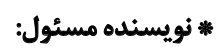

دكتر معصومه افسا

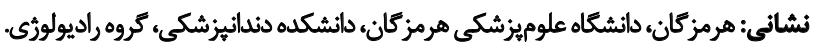

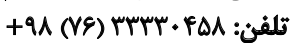
بهت الكترونيكي: masomeh.afsa@hums.ac.ir 

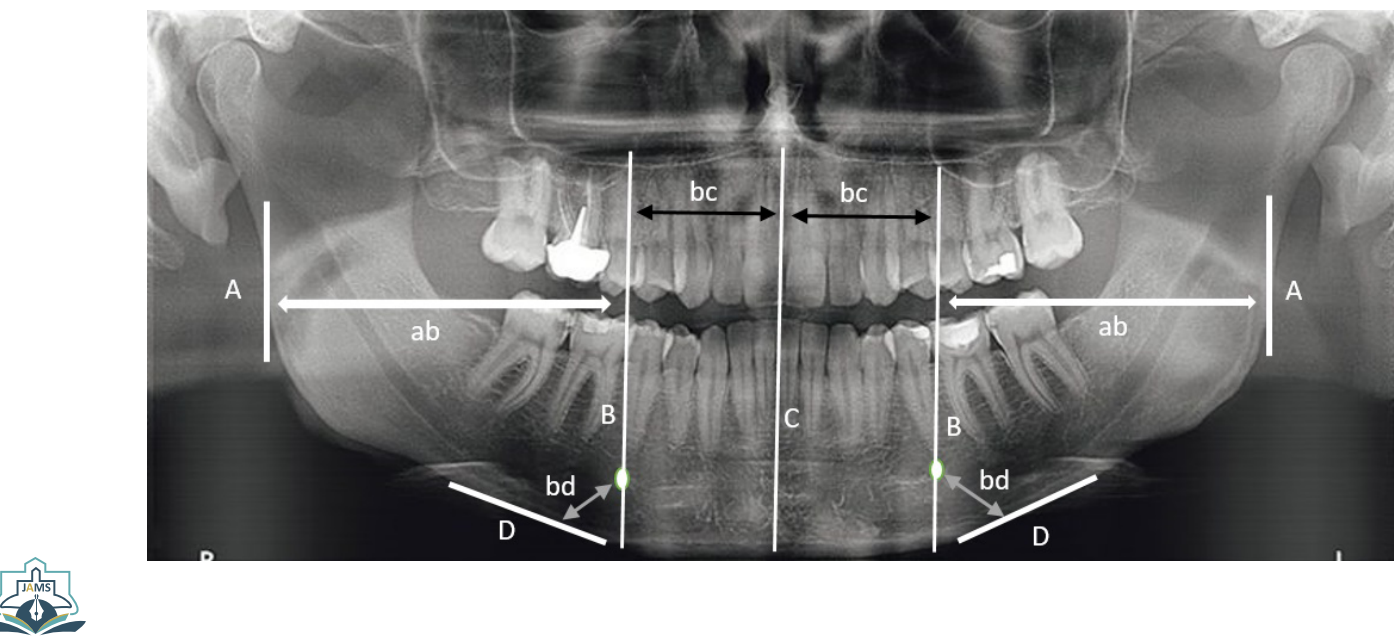

تصوير ا. نحوه آناليز و بررسى تصاوير راديوكرافى تهيهشده از بيماران

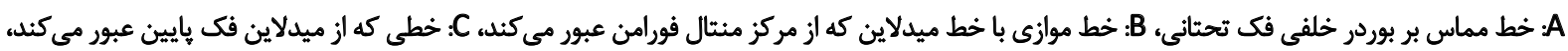

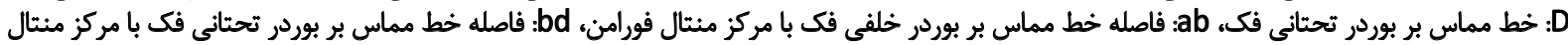

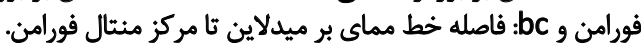

\section{مواد ورش}

مطالعه حاضر يك مطالعه مشاهدالى مقطعى است. براى انجام

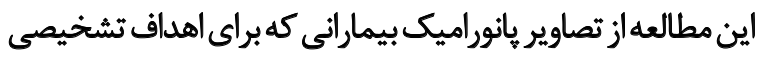

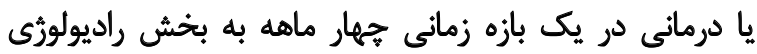

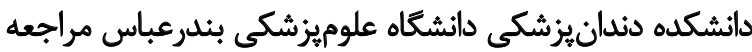

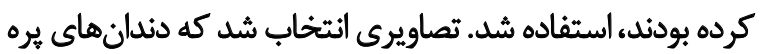

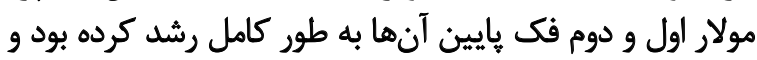
فاقد هركونه ضايعه راديولوسنت بواين آنماند طوند

همجنين اين دندانها در مرحله دائمى قرار داشته و بيمار

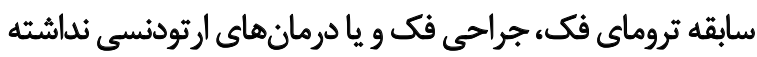

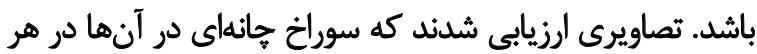

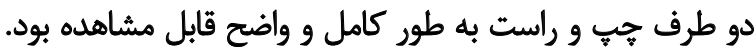
درنهايت تعداد • fo تصوير مطالعه و بررسى شداند.

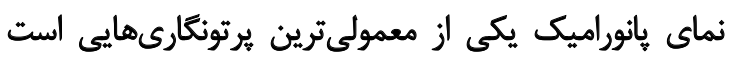

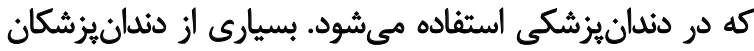

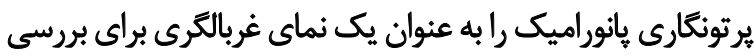

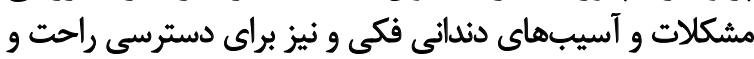

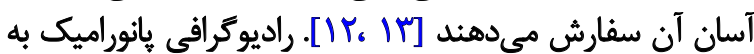

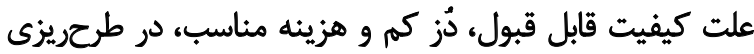

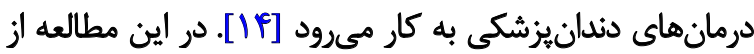

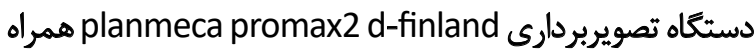
با نمايشكَر لِّتاب Asus جهت تهيه تصاوير استفاده شد.

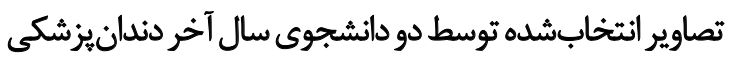

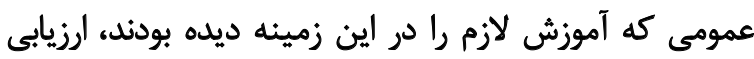

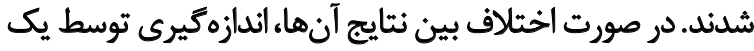

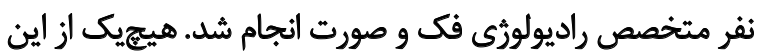

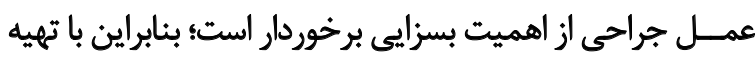

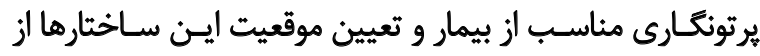

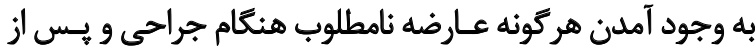

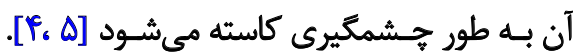

امروزه اعمال جراحى بسيارى در ناحيه جلويى فـى تحتانى

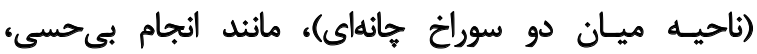

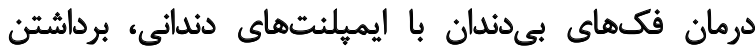

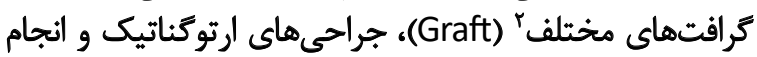

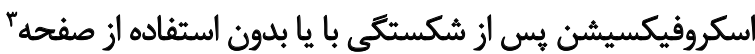

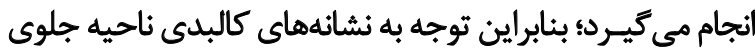

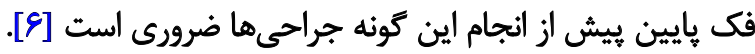

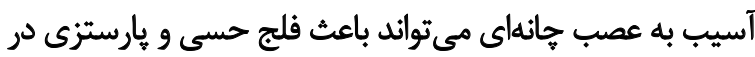

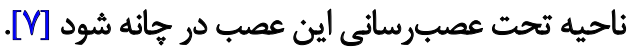
جائيدى و همكاران در مطالعهاى بيان كردند كه موقعيت سوراخ إناخ

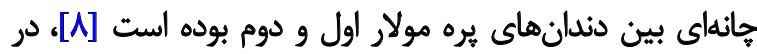

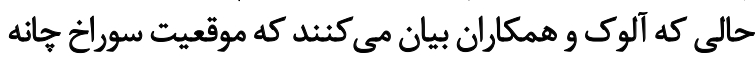

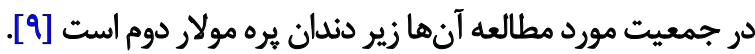

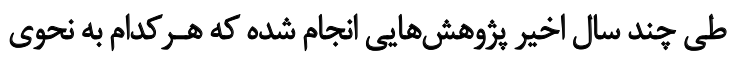

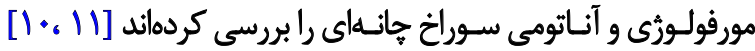

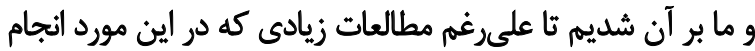

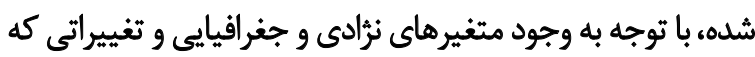

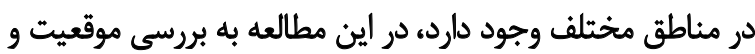

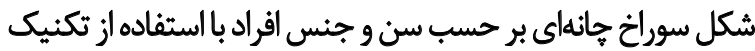
راديوكرافى يانوراميك در بخشى از كشور بيردازيه.

2. Screw fixation

3. Plate 
جدول ا. ميائكين فواصل قدامى خلفى سوراخ جانهاي تا ميدلاين، بوردر خلفى و بوردر تحتائى مثديبل در كروههاي سنى مورد مطالعه

\begin{tabular}{|c|c|c|c|c|c|c|c|c|c|c|}
\hline \multirow{3}{*}{$\begin{array}{l}P \\
\text { के }\end{array}$} & \multirow{3}{*}{ راست } & \multicolumn{8}{|c|}{ ميانغين土|نحراف معيار } & \multirow{3}{*}{ مثغيرها } \\
\hline & & \multicolumn{2}{|c|}{ كروه سنى د } & \multicolumn{2}{|c|}{ كروه سنى ع } & \multicolumn{2}{|c|}{ كروه سنى ب } & \multicolumn{2}{|c|}{ ئروه سنى الف } & \\
\hline & & هب & راست & جي" & راست & جي & راست & هูب & راست & \\
\hline.$/ 4 \Delta$ & .195 & $r W / Y \pm T / * \Lambda$ & $r V \pm r / \Lambda \Delta$ & $r \varepsilon / \mathscr{R} \mid \pm r / \Lambda 1$ & WVRADT/QS & $r g / 1 \pm T / R A$ & $\mathrm{TW} / \mathrm{T} \pm \mathrm{r} / \mathrm{NF}$ & $r e / T \pm T / K g$ & $r \Delta / \Delta \pm T / Q \Delta$ & فاصله تا ميدلاين \\
\hline .1 .9 &.$/ T \Lambda$ & PNE $\pm F / T$ & $r N q \pm F / T A$ & $F N M \pm F / \triangle A$ & Pq/RAtp/ge & PNREIA/NT & 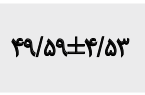 & Peleq & $P N \cdot \mid \pm d / M A$ & فاصله تا كنار \\
\hline$+1+\%$ & .10 .1 & $1 . / 91 \pm 1 / T r$ & $\mid 1 / n \pm 1 / \pi$ & $1+/ 8 \mu \pm 1 / \Delta q$ & $1 . / N \pm V / \Delta S$ & $1+/ F q \pm 1 / 0$ & $1+/ T H \pm V / \Delta F$ & $q / Q \pm 1 / V \Delta$ & $q / q \pm 1 / \pi$ & فاصله تا كنار \\
\hline
\end{tabular}

جدول Y. ميانكين فواصل قدامى خلفى سوراخ جانهاى تا ميدلاين، بوردر خلفى و بوردر تحتانى منديبل بر حسب جنس بيماران

\begin{tabular}{|c|c|c|c|c|c|c|}
\hline \multirow{3}{*}{$\begin{array}{l}\mathbf{P} \\
\stackrel{\forall}{*}\end{array}$} & \multirow{3}{*}{ راست } & \multicolumn{4}{|c|}{ ميانكين ||نحراف معيار } & \multirow{3}{*}{ متغيرها } \\
\hline & & \multicolumn{2}{|c|}{ ji } & \multicolumn{2}{|c|}{ مرد } & \\
\hline & & حै" & راست & ه & راست & \\
\hline$+1 .+9$ & $+1 .+1$ & $r \Delta / q \pm \Gamma / . r$ & $r E / M \pm r / q q$ & $r E / F A \pm r / 1 q$ & $W / g r \pm r / 19$ & قاصله تا ميدلاين \\
\hline$<\cdot 1 \cdots+1$ & $<\cdot(\cdots)$ & $P V / V \& \pm \Delta / \% T$ & ENTEET/KI & $P q / A \pm F / R Y$ & $\Delta \cdot / \Delta \pm \varphi / \varepsilon \Delta$ & فاصله تا بوردر خلفى \\
\hline$<+1+\cdots+1$ & $<+1+\cdots+1$ & $1 . / * \pm 1 /{ }^{\prime} 1$ & $\checkmark / 4 \pm 1 / r^{\prime} \Delta$ & $\| / \cdot A \pm V / \Delta \Delta$ & $W / / F \pm 1 / \Delta$ & فاصله تا بوردر تحتانى \\
\hline
\end{tabular}

\section{(sims}

فكى تحتانيى در دو طرف (فاصله عمودى بين مركز سوراخ جانه ايى با خطى كه مماس با لبه خلفى فك يايين ترسيم شده). 9. موقعيت قدامي خلفي سوراخ جانهاي بر اساس موقعيت

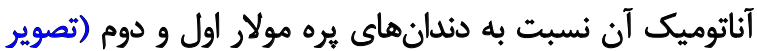
شيماره (1) (1) دادهاي به دست آمده با نرمافزار SPSS نسخه

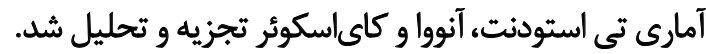

bodigl

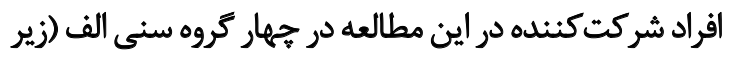
.

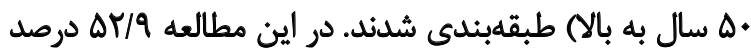

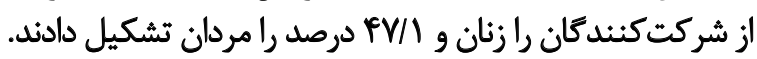
ا. فاصله قدامي خلفي سوراخ جانهاي تا ميدلاين و كنار خلفي

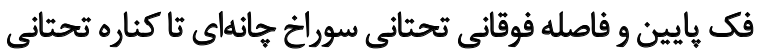
فكى بر حسب سن بيماران:

اندازهكيرى موقعيت قدامى خلفي سوراخ جانهاي ثا ميدلاين

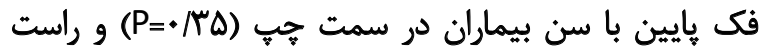

افراد از هدف مطالعه اطلاعى نداشتئد و تنهيا اندازهكيرى ها رانجام

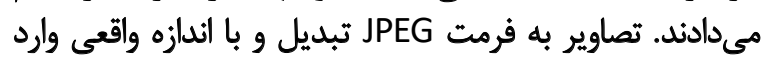

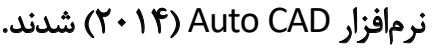
با استفاده از امكانات اين ترمافزار، خطوطي مماس بالبه تحتاني

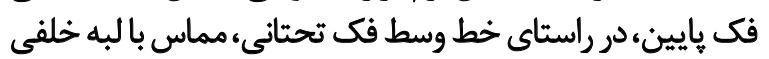
فك تحتانى و همجينين خطى موازى با ميدلاين كه از مركزئ

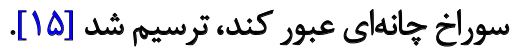
تصاوير يانوراميك براي تجزيه و تحليل موارد زير ارزيابي شدند: ا.شكل سوراخ هانهاى در هر طرف (بيضى يا كرد). r. اندازه سوراخ خانهاى در هر طرف. r. موقعيث فوقانى تحتائى سوراخ جانهاي نسبت به لبه تحتانى

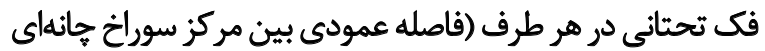
با خط مماس با لبه تحتانى فك تحتائى). f. موقعيت قدامي خلفي سوراخ جانهاي نسبت به خط ميدلاين

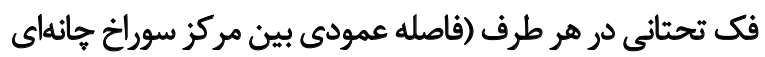

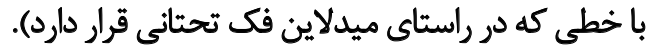
ه. موقعيت قدامي خلفي سوراخ خانهاى نسبت به لبه خلفي 
جدول r. شكل سوراخ جانهاى در راديوكرافى يانوراميك بر حسب جنس بيماران

\begin{tabular}{|c|c|c|c|c|}
\hline \multicolumn{4}{|c|}{ فراوانى (درصد) } & \multirow{3}{*}{ 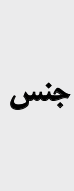 } \\
\hline \multicolumn{2}{|c|}{ شكل سوراخ خانهالى در سمت جِب فك بايين } & \multicolumn{2}{|c|}{ شكل سوراخ جانهايى در سمت راست فك بايين } & \\
\hline بيضى & 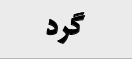 & بيضى & ترك & \\
\hline IAS $(N N T)$ & $r V(I T / M)$ & $M+(A F / q)$ & $\pi(\mid \Delta /)$ & مرد \\
\hline r.q (A\&/q) & $\pi r\left(I r / T^{*}\right)$ & $(r \cdot M F / \Delta)$ & $r V(1 \otimes / \Delta)$ & زن \\
\hline \multicolumn{2}{|c|}{. Ar } & \multicolumn{2}{|c|}{$+/ 19$} & $\mathrm{P}$ \\
\hline
\end{tabular}

جدول f. شكل سوراخ جانهاى در راديوكرافي بانوراميك بر حسب سن بيماران

\begin{tabular}{|c|c|c|c|c|}
\hline \multicolumn{4}{|c|}{ فراوانى (درصد) } & \multirow{3}{*}{ 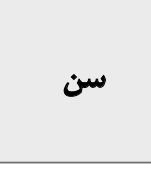 } \\
\hline \multicolumn{2}{|c|}{ شكل سوراخ هانهاي در سمت هبب } & \multicolumn{2}{|c|}{ شكل سوراخ جانهاي در سمت راست } & \\
\hline بيضى & كرد & بيضى & ترد & \\
\hline$r \Delta(A r / \pi)$ & $\Delta(I E M)$ & $\pi r(N \& / 9)$ & $V(\pi / r)$ & كروه سنى الف \\
\hline $\operatorname{rra}(\Lambda)$ & $M(M)$ & $r+(N=1)$ & $\operatorname{rr}(1 Y / 9)$ & كروه سنى ب \\
\hline $118(A V / q)$ & $18(1 \mathrm{~T} / 1)$ & $m(N=1)$ & $r(1 \Delta / 9)$ & كروه سنى ج \\
\hline \multirow[t]{2}{*}{$10\left(M /{ }^{*}\right)$} & $8(T N E)$ & $\operatorname{Ir}(\lambda))$ & $P(19)$ & كروه سنى د \\
\hline & & \multicolumn{2}{|c|}{ / } & $\mathrm{P}$ \\
\hline
\end{tabular}

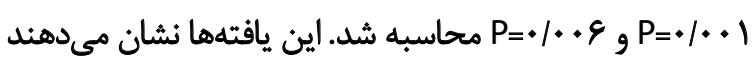

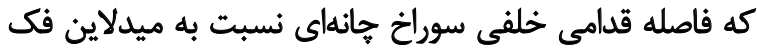

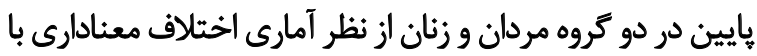
هم دارند (جدول شماره ؟). در بررسى ارتباط بين موقعيت قدامى خلفى سوراخ خانهاى

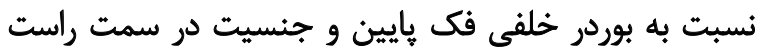

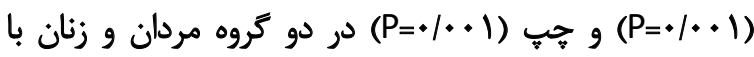
همديكر اختلاف معنادارى داشتند (جدول شماره Y). در بررسى ارثباط بين موقعيت قدامى خلفى سوراخ خانهاي

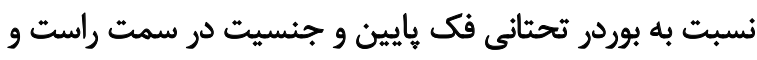

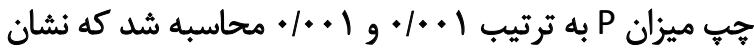

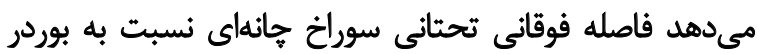

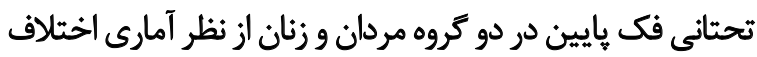

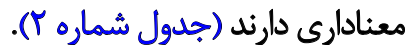
ז. شكل سوراخ خانهاى در راديوكرافى هانوراميك بر حسب

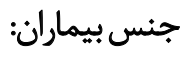
در بررسى ارتباط بين شكل سوراخ خانهاى و جنسيت در
(P=•/Af) تفاوت معنادارى با يكديكر نداشتند (جدول شمال شماره (). در بررسى ارتباط بين موقعيت قدامى خلفى سوراخ خانهاي

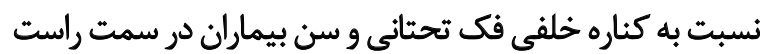

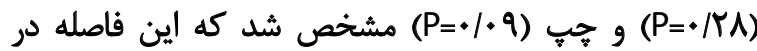

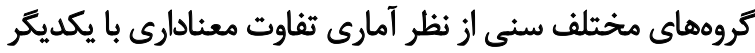

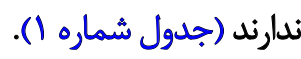

همجنين در بررسى ارتباط بين موقعيت فوقانى تحتانى سوراخ

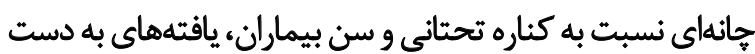

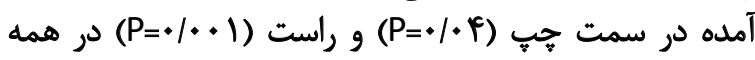

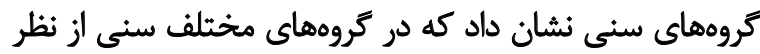

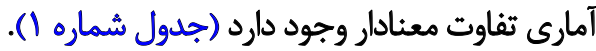

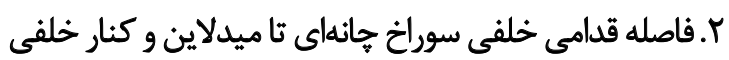

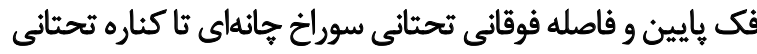
فك بر حسب جنسيت بيماران:

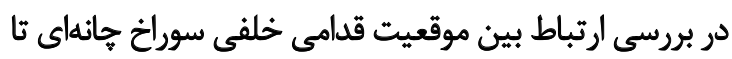

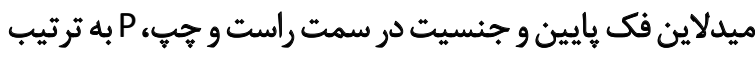


حيدر آباد هند بين دندانهاى يره مولار اول و دوم است، همجينين

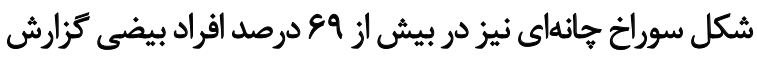

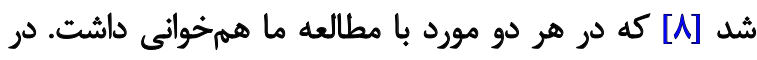

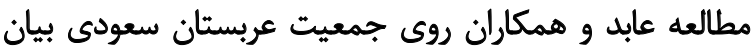

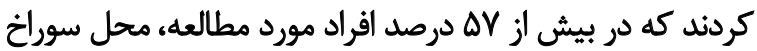

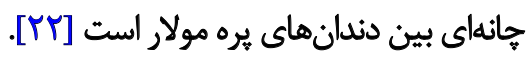

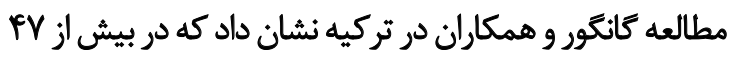

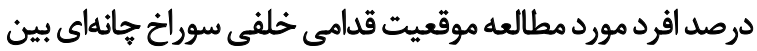

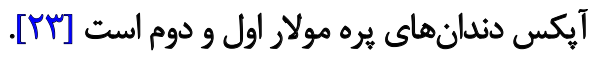

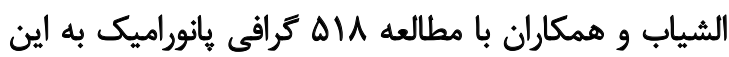

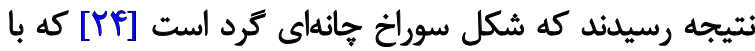

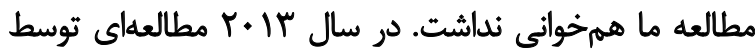

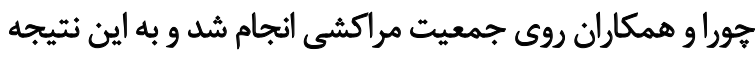

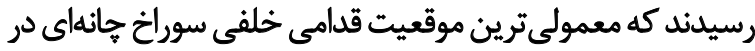

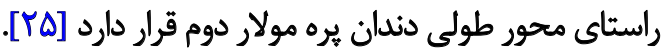
علاوه بر اين، در مطالعه ديكرى كه توسط جامداد و همكاران

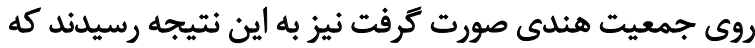

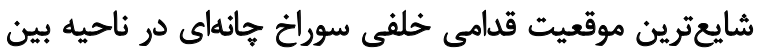

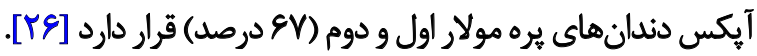

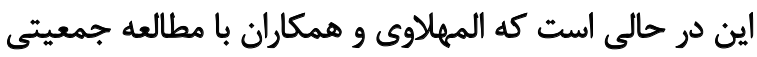

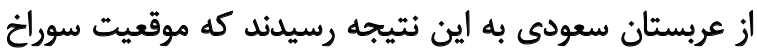

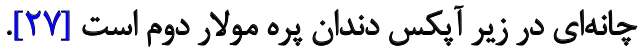

همجينين در مطالعه ديكرى كه در كشور عراق توسط محمد

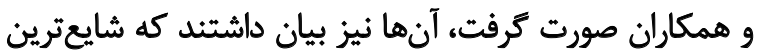

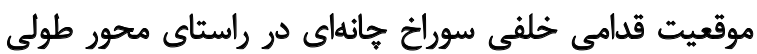

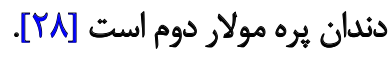

در ايران نيز مطالعات متعددى توسط محققان در اين مورد

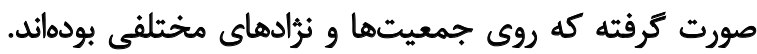

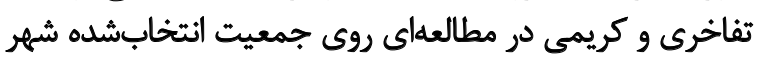

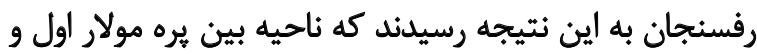

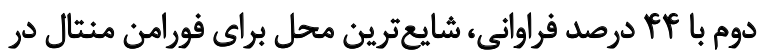

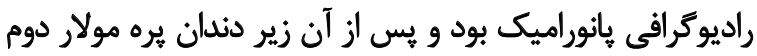

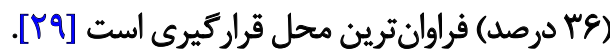

حقانى فرو همكاران روى جمعيت منتخب در شهر بابل صورت

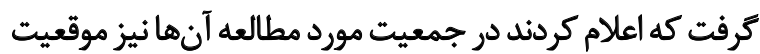

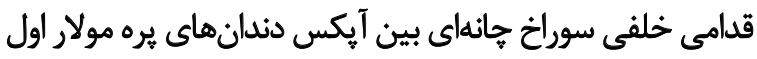

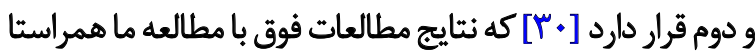

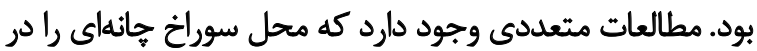

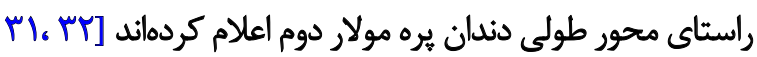
9] كه نتايج آنها با مطالعه ما همخواني نداشتئند. در مطالعه ماء موقعيت قدامى خلفى سوراخ خانهاى نسبت به

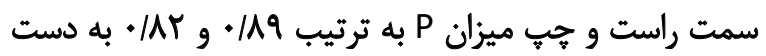

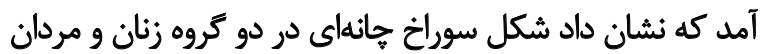

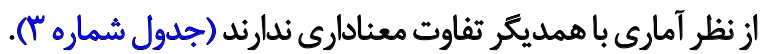
f. شكل سوراخ جانهاى در راديوكرافى پانوراميك بر حسب

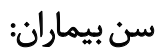

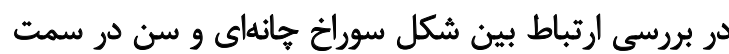

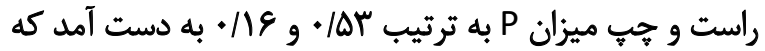

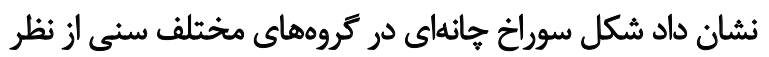
آمارى با همديكر تفاوت معنادارى ندارند (جدول شمان شماره ثأ). ثैب

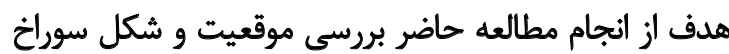

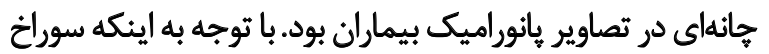

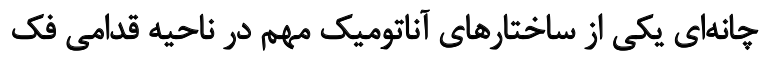

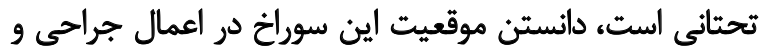

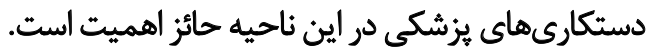

در اين مطالعه به منظور تعيين موقعيـت آناتوميك و شكل

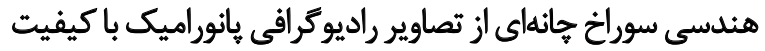

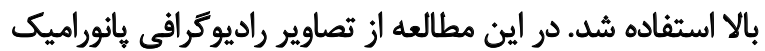

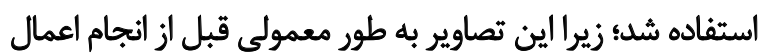

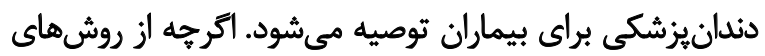

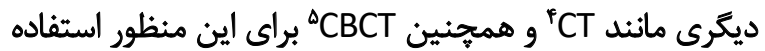
ميشود كه دقت بالاترى نيز دارند.

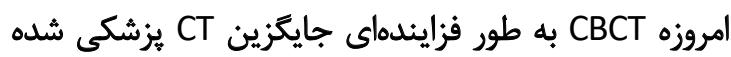

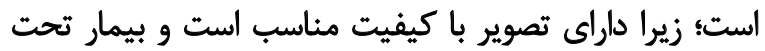

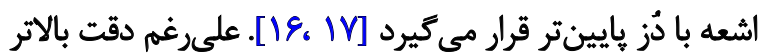

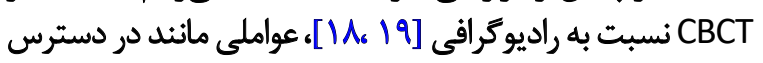

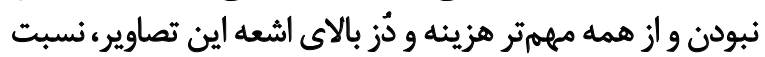

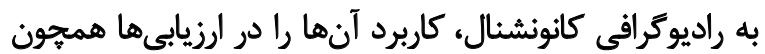

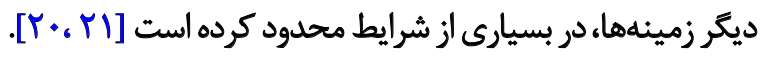

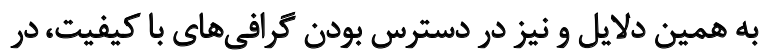
مطالعه حاضر از تصاوير راديوكرافى استفاده شد.

نتايج مطالعه حاضر نشان مي دهد كه به لحاظ موقعيت قدامي

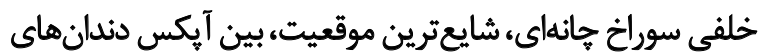

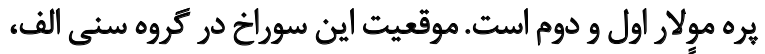

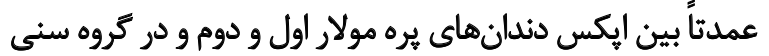

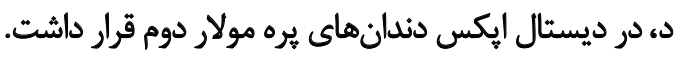
در مطالعه جاييدى و همكاران مشخص شد كه موقعيت سوراخ

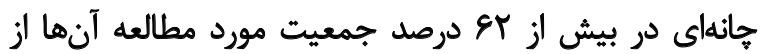

4. Computed Tomography

5. Cone Beam Computed Tomography 


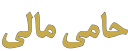

اين مقاله بركرفته از رساله دكترى عمومى در دندانيزشكى دئي

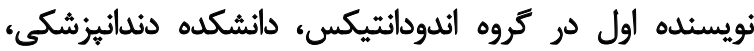
دانشكاه علوميزشكى شهيد صدوقى يزد است.

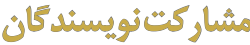

مفهومسازى: معصومه افسا، روش شناسى و اعتبارسنجى: امين قنبرنزاد، تحقيق، بررسى و نكارش: حمزه اكران.

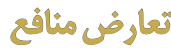

بنابر اظهار نويسندكان هيجگ ينهنه تعارض منافعى در خصوص

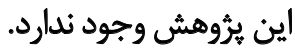

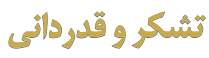

از همكارى دانشكاه علوميزشكى هرمزكان و كاركنان و همكاران

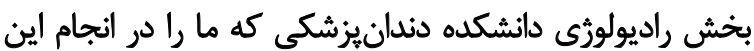

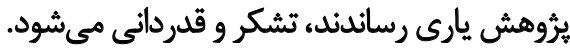

خط ميدلاين فك تحتاني، در سمت راست و هبٍ به ترتيب

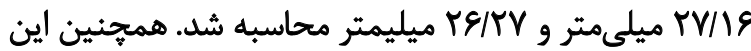

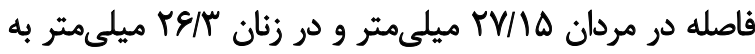
دست آمد كه بين زنان و مردان اختلاف معناداري مشاهده شئ مئل در مطالعه ناناياكارا و همكاران در سريلانكا روى جمجمههاي

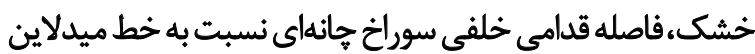

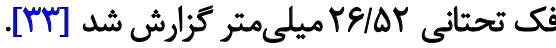
در مطالعه حاضرء از نظر موقعيت قدامى خلفى سوراخ خانهاي

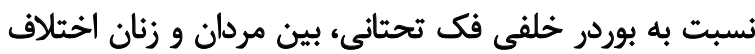

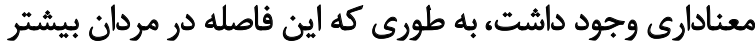

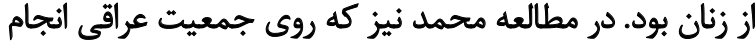

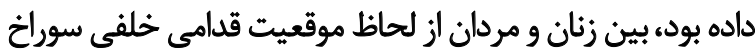

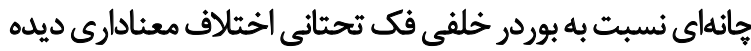

شد [Y]

از نظر موقعيت فوقانى تحتانى سوراخ جانهاي نسبت به بوردر

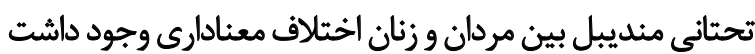

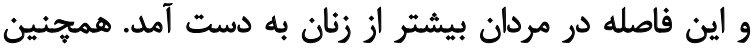

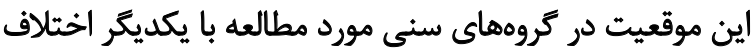

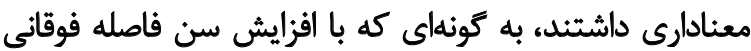

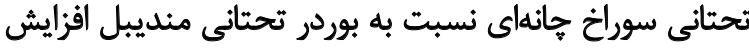

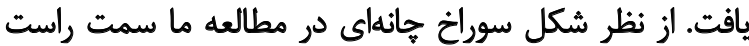

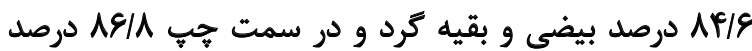

$$
\text { بيضى و بقيه ترد بودي. }
$$

در مطالعه سريواستاى و سينگ كه روى جمجمهلهاى خشك

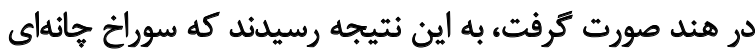

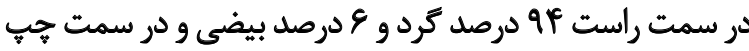

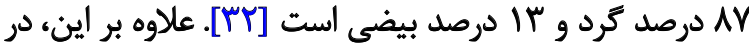

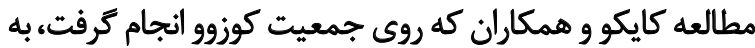

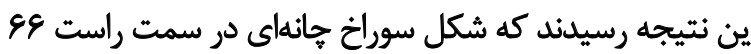

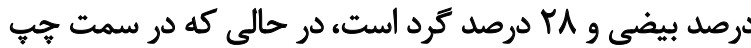

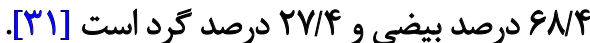
با توجه به مطالعه حاضر و مطالعات ديكر، شكل سوراخ

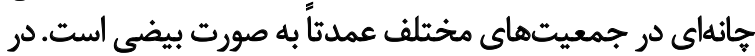

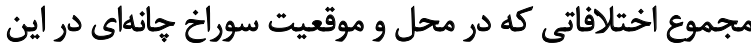

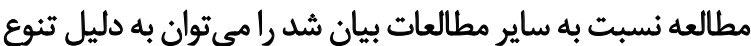

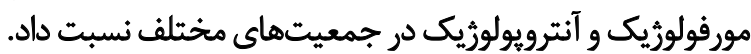

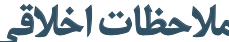

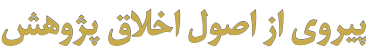
اين مطالعه با كد اخلاق IR.HUMS.REC.1394.189 به تصويب كميته اخلاق معاونت يروهشى دانشكاه علوميزشكى هرمزكان رسيد. 


\section{References}

[1] Iwanaga J, Saga T, Tabira Y, Nakamura M, Kitashima S, Watanabe K, et al. The clinical anatomy of accessory mental nerves and foramina. Clin Anat. 2015; 28(7):848-56. [DOI:10.1002/ca.22597] [PMID]

[2] Loudon J. Beware the mental foramen. Br Dent J. 2011; 210(7):293. [DOI:10.1038/sj.bdj.2011.249] [PMID]

[3] Udhaya K, Saraladevi KV, Sridhar J. The morphometric analysis of the mental foramen in adult dry human mandibles: A study on the South Indian population. J Clin Diagn Res. 2013; 7(8):1547-51. [DOI:10.1038/ sj.bdj.2011.249] [PMID]

[4] Al-Juboori MJ, Hua CM, Yuen KY. The importance of the mental foramen location detection by using different radiographic technique: Mini review. International J Med Imaging. 2014; 2(3):63-8. [DOI:10.11648/j. ijmi.20140203.14]

[5] Zmysłowska-Polakowska E, Radwański M, Łęski M, Ledzion S, ŁukomskaSzymańska M, Polguj M. The assessment of accessory mental foramen in a selected polish population: A CBCT study. BMC Med Imaging. 2017; 17(1):17. [DOI:10.1186/s12880-017-0188-6] [PMID] [PMCID]

[6] Aminoshariae A, Su A, Kulild JC. Determination of the location of the mental foramen: A critical review. J Endod. 2014; 40(4):471-5. [DOI:10.1016/j.joen.2013.12.009] [PMID]

[7] Chappidi V, Swapna LA, Dheeraj V, Nikitha GR, Kanakagiri M. Evaluation of morphometric variations in mental foramen and prevalence of anterior loop in South Indian population: A CBCT study. J Indian Acad Oral Med Radiol. 2019; 31(2):134-9. [DOI:10.4103/jiaomr.jiaomr_219_18]

[8] Alok A, Singh ID, Singh S. Evaluation of styloid process in bareilly population on digital panoramic radiographs. J Indian Acad Oral Med Radiol. 2016; 28(4):381-5. [DOI:10.4103/0972-1363.200623]

[9] Cakur B, Dagistan S, Shahin A, Haroli A, Yilmaz A. Reliability of mandibular cortex index and mandibular bone mineral density in the detection of osteoporotic women. Dentomaxillofac Radiol. 2009; 35(5):255-61. [DOI:10.1259/dmfr/22559806] [PMID]

[10] Cakur B, Sahin A, Dagistan S, Altun O, Caglayan F, Miloglu O, et al. Dental panoramic radiography in diagnosis of osteoporosis. J Int Med Res. 2008; 36(4):792-9. [DOI:10.1177/147323000803600422] [PMID]

[11] Mraiwa N, Jacobs R, van Steenberghe D, Quirynen M. Clinical assessment and surgical implications of anatomic challenges in the anterior mandible. Clin Implant Dent Relat Res. 2003; 5(4):219-25. [DOI:10.1111/j.1708-8208.2003.tb00204.x] [PMID]

[12] Jacobs R, Mraiwa N, vanSteenberghe D, Gijbels F, Quirynen M. Appearance, location, course, and morphology of the mandibular incisive canal: An assessment on spiral CT scan. Dentomaxillofac Radiol. 2002; 31(5):322-7. [DOI:10.1038/sj.dmfr.4600719] [PMID]

[13] Tözüm TF, Taguchi A. Role of dental panoramic radiographs in assess ment of future dental conditions in patients with osteoporosis and periodontitis. N Y State Dent J. 2004; 70(1):32-5. [PMID]

[14] Niknami M, Esmaeeli F, Nazer A. [Radiographic evaluation of location and shape of mental foramen in selective Iranian Population (Persian)] Res Dent Sci. 2011; 8(2):102-8. http://jrds.ir/article-1-46-en.pdf

[15] Ziegler CM, Woertche R, Brief J, Hassfeld S. Clinical indications for digital volume tomography in oral and maxillofacial surgery. Dentomaxillofac Radiol. 2002; 31(2):126-30. [DOI:10.1038/sj.dmfr.4600680] [PMID]

[16] Miracle AC, Mukherji SK. Conebeam CT of the head and neck, part 1: Physical principles. AJNR Am J Neuroradiol. 2009; 30(6):1088-95. [DOI:10.3174/ajnr.A1653] [PMID] [PMCID]
[17] Muinelo-Lorenzo J, Suárez-Quintanilla JA, Fernández-Alonso A, VarelaMallou J, Suárez-Cunqueiro MM. Anatomical characteristics and visibility of mental foramen and accessory mental foramen: Panoramic radiography vs. Cone beam CT. Med Oral Patol Oral Cir Bucal. 2015; 20(6) e707-14. [DOI:10.4317/medoral.20585] [PMID] [PMCID]

[18] Naitoh M, Yoshida K, Nakahara K, Gotoh K, Ariji E. Demonstration of the accessory mental foramen using rotational panoramic radiography compared with cone-beam computed tomography. Clin Oral Implants Res. 2011; 22(12):1415-9. [DOI:10.1111/j.1600-0501.2010.02116.x] [PMID]

[19] Sukovic P. Cone beam computed tomography in craniofacial imaging Orthod Craniofac Res. 2003; 6 (Suppl 1):31-6. [DOI:10.1034/j.16000544.2003.259.x] [PMID]

[20] Falk A, Gielen S, Hauser L. CT data acquisition as a basic for modern diagnosis and therapy in Maxillofacial surgery. Int J Oral Maxillofac Surg. 1995; 24(1):69-75. [DOI:10.1016/S0901-5027(05)80865-3]

[21] Abed HH, Bakhsh AA, Hazzazi LW, Alzebiani NA, Nazer FW, Yamany I, et al. Anatomical variations and biological effects of mental foramen position in population of Saudi Arabia. Dentistry. 2016; 6(4):373. https://www.researchgate.net/profile/Abdulaziz-Bakhsh/publication/301898976_Anatomical_Variations_and_Biological_Effects_ of_Mental_Foramen_Position_in_Population_of_Saudi_Arabia/ links/572c605808ae0a72918a4215/Anatomical-Variations-and-Biological-Effects-of-Mental-Foramen-Position-in-Population-of-SaudiArabia.pdf

[22] Gungor K, Ozturk M, Semiz M, Brooks SL. A radiographic study of location of mental foramen in a selected Turkish population on panoramic radiograph. Coll Antropol. 2006; 30(4):801-5. [PMID]

[23] Alshayyab MH, Alsoleihat F, DarOdeh NS, Ryalat S, Baqain ZH. The mental foramen I: Radiographic study of the anteriorposterior position and shape in Iraqi population. Int J Morphol. 2015; 33(1):149-57 [DOI:10.4067/S0717-95022015000100024]

[24] Chkoura A, El Wady W. Position of the mental foramen in a Moroccan population: A radiographic study. Imaging Sci Dent. 2013; 43(2):71-5. [DOI:10.5624/isd.2013.43.2.71] [PMID] [PMCID]

[25] Kumar V, Hunsigi P, Kaipa BR, Reddy R, Ealla KK, Kumar CB, Prasanna MD. Radiographic localization of mental foramen in Northeast and South Indian ethnic groups of Indian population. J Contemp Dent Pract. 2014; 15(6):766-9. https://www.thejcdp.com/doi/pdf/10.5005/jpjournals-10024-1614

[26] Al-Mahalawy H, Al-Aithan H, Al-Kari B, Al-Jandan B, Shujaat S. Determination of the position of mental foramen and frequency of anterior loop in Saudi population: A retrospective CBCT study. Saudi Dent J. 2017 29(1):29-35. [PMID] [PMCID]

[27] Muhammad DA. Anteroposterior position of the mental foramen on panoramic radiographs in Sulaimani population. Kurdistan Acad J. 2009; 8:9-16. [Link not Found]

[28] Tafakhori Z, Karimi M. [Evaluation of horizontal position of mental foramen in relation to mandibular premolar in the panoramic radiograph in Rafsanjan City in 2014 (Persian)]. J Rafsanjan Univ Med Sci. 2016 14(11):913-22. http://journal.rums.ac.ir/article-1-2633-en.html

[29] Haghanifar S, Rokouei M. Radiographic evaluation of the menta foramen in a selected Iranian population. Indian J Dent Res. 2009; 20(2):150-2. [DOI:10.4103/0970-9290.52886] [PMID]

[30] Kqiku L, Sivic E, Weiglein A, Städtler P. Position of the mental foramen: An anatomical study. Wien Med Wochenschr. 2011; 161(9-10):272-3. [DOI:10.1007/s10354-011-0898-2] [PMID] 
[31] Singh, R, Srivastav, AK. Study of position, shape, size and incidence of mental foramen and accessory mental foramen in Indian adult human skulls. Int J Morphol. 2010; 28(4):1141-6. [DOI:10.4067/S071795022010000400025]

[32] Prabodha LB, Nanayakkara BG. The position, dimensions and morphological variations of mental foamen in mandibles. Galle Med J. 2006; 11(1):13-5. [DOI:10.4038/gmj.v11i1.1111]

[33] Nanayakkara D, Sampath H, Manawaratne R, Peiris R, Vadysinghe A, Arambawatte K. Positional variation and localization of the mental foramen. MOJ Anat Physiol. 2018; 5(1):39-44. [DOI:10.15406/mojap.2018.05.00162] 
This Page Intentionally Left Blank 